\title{
Augmenting Sequence Enumeration with String-Rewriting for Requirements Analysis and Behavioral Specification
}

\author{
Lan Lin $^{1}$, Jesse H. Poore ${ }^{2}$, Robert Eschbach ${ }^{3}$, \\ Robert M. Hierons ${ }^{4}$, and Christopher Robinson-Mallett ${ }^{5}$ \\ 1 Department of Computer Science, Ball State University, Muncie, IN, USA \\ llin4@bsu.edu \\ 2 Department of Electrical Engineering and Computer Science, \\ University of Tennessee, Knoxville, TN, USA \\ poore@eecs.utk.edu \\ 3 ITK Engineering AG, Herxheim, Germany \\ robert.eschbach@itk-engineering.de \\ 4 School of Information Systems, Computing and Mathematics, Brunel University, \\ Uxbridge, Middlesex, UK \\ rob.hierons@brunel.ac.uk \\ 5 Berner \& Mattner Systemtechnik GmbH, Berlin, Germany \\ christopher.robinson-mallett@berner-mattner.com
}

\begin{abstract}
Sequence enumeration is a method for deriving a system model based on informal requirements. Under sequence enumeration, stimulus (input) sequences are considered in a breadth-first manner, with the expected system response to each sequence given. Not all sequences of stimuli are considered since a sequence need not be extended if either it is illegal (it cannot be applied in practice) or it can be reduced to another sequence previously considered (the sequences take the system to the same state). Sequence enumeration is mostly a manual process, which leads to a model that can be used as the basis for automation. This paper describes a method, based on string-rewriting, that automates parts of sequence enumeration. This automation has the potential to reduce both the cost and time involved in sequence enumeration but also to reduce the scope for human error. In addition to outlining this method, we discuss our experiences in applying it to four case studies.
\end{abstract}

Keywords: software specification, sequence-based specification, sequence enumeration, string-rewriting, requirements engineering.

\section{Introduction}

Software development often starts with all sorts of functional requirements. They are generally written in a natural language, and contain ambiguities, omissions, inconsistencies, and errors. All these problems need to be resolved and the requirements need to be converted into a precise specification at an early stage

V. Cortellessa and D. Varró (Eds.): FASE 2013, LNCS 7793, pp. 179-193, 2013.

(C) Springer-Verlag Berlin Heidelberg 2013 
in the development life cycle. The specification is important for later phases including code development, testing, as well as functional formal verification.

The sequence-based specification method [12 19 21] was developed for this purpose, providing a systematic way to convert imprecise and informal requirements into precise software specifications, through sequence enumeration. In this process, the developer or domain expert considers sequences of stimuli in a breadthfirst manner, where sequences of a given length are examined in lexicographic order. For each such sequence, the developer gives the last output produced in response to the sequence. There are two situations in which a sequence need not be further extended: either it is illegal (it cannot be applied in practice) or it reaches a system state equivalent to one reached by a shorter or lexicographically earlier sequence. Sequence enumeration stops when no sequence needs to be extended and results in a table of sequences that defines a Mealy machine.

A significant benefit of sequence enumeration is that it results in a formal specification but without requiring the developer to use a formal notation. This specification can form the basis for other activities, such as automated (modelbased) testing. The specification can also be automatically analyzed to determine whether it has certain expected, or desirable properties; this can result in requirements errors being found. The number of sequences considered is also equal to the number of transitions of the Mealy machine that represents the requirements and so the cost of sequence enumeration can be seen as being linear in terms of the complexity of the requirements. However, automation has the potential to allow sequence enumeration to scale further. This paper describes a method in which string-rewriting rules are used in order to automate the analysis of some sequences. This reduces the number of sequences that have to be considered, and thus can reduce both the cost of sequence enumeration and the potential for human error. We also explain how possible rules can be deduced from previous reductions; potentially these might be provided to the developer who can choose to accept or reject them. The overall approach has been implemented in our enumeration tool [18] and we describe the result of applying this tool to four case studies.

This work was motivated by the various observed patterns in analyzing enumerated sequences in field applications [2, 4, 10, 21. With the new theory and accompanying tool support, more equivalence declarations can be handled automatically and consistently leading to fewer human errors. The result is an enhanced enumeration process. The method treats discrete systems, and systems modeled as discrete based on abstractions of events.

This paper is structured as follows. In Section 2 we describe sequence enumeration and a running example. Section 3 describes the proposed method that uses string-rewriting in sequence enumeration, while Section 4 outlines our experience in using this method with four case studies. Section 5 summarizes related work. Finally, Section [6] draws conclusions and discusses future work. 


\section{Developing a Behavioral Specification through Sequence Enumeration}

Sequence-based specification [12, 19 21] uses a technique called sequence enumeration to discover the behavior of the software. The resulting specification can be converted to a state machine for implementation and testing.

To apply the method, one first identifies a system boundary that defines what is inside and outside the software-intensive system. This usually consists of a list of interfaces between the system and the environment of the software.

Throughout the paper we will use an automobile mirror electronic control unit (ECU) as a running example. The example was taken from 2 and modified to fit the page limit. The original requirements are collected in Table 1, with each sentence numbered (tagged) for easy reference.

Table 1. Requirements for the driver side car mirror ECU

\begin{tabular}{l|l}
\hline Trace Tag & Requirement \\
\hline 1 & $\begin{array}{l}\text { There is a switch that toggles for adjustment of either the driver side or } \\
\text { the passenger side mirror. } \\
\text { The driver side electronic control unit (ECU) initializes when the car } \\
\text { key is in start position. } \\
\text { The driver side ECU processes inputs from position sensors and users. } \\
\text { The driver side ECU produces outputs to actuators and sends messages } \\
\text { to other ECUs. }\end{array}$ \\
5 & $\begin{array}{l}\text { Control area network (CAN) bus used for communication among ECUs. } \\
\text { Signals for the passenger side mirror are put on the CAN bus and sent } \\
\text { to the passenger side ECU. } \\
\text { Each mirror can be adjusted vertically and has extreme up and down } \\
\text { positions. } \\
\text { Each mirror can be adjusted horizontally and has extreme inward and } \\
\text { outward positions. } \\
\text { If requested movement cannot be made because the mirror is already } \\
\text { in an extreme position, an error message is generated and sent via the } \\
\text { CAN bus. }\end{array}$ \\
9 &
\end{tabular}

We identify a system boundary in Figure 1, and list the interfaces in Table 2 with traces to the tagged requirements. From the interfaces one further collects stimuli and responses. Stimuli refer to events (inputs, interrupts, invocations) in the environment that can affect system behavior. Responses refer to system behaviors observable in the environment. The stimuli and responses for the car mirror ECU are identified in Table 3 and Table 4.

We use $S$ and $R$ to denote the stimulus set and the response set, respectively. As the name "sequence enumeration" suggests, one explicitly enumerates all finite stimulus sequences from $S^{*}$ (representing scenarios of use), first in increasing order of length, and within the same length lexicographically. We use $\lambda$ 


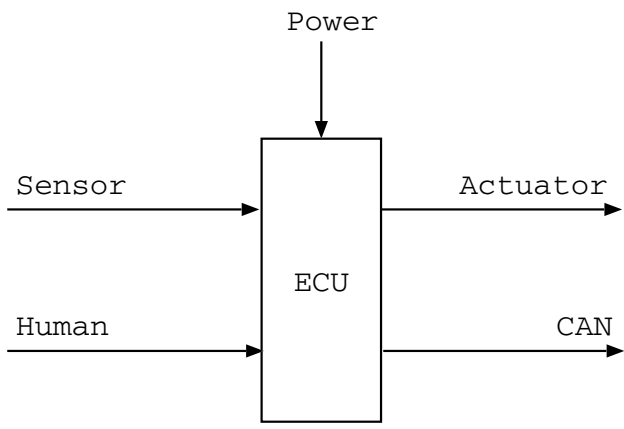

Fig. 1. A system boundary for the driver side car mirror ECU

Table 2. Interfaces for the driver side car mirror ECU

\begin{tabular}{l|l|l}
\hline Interface & Description & Trace \\
\hline Actuator & The actuators & 4 \\
CAN & The CAN bus & $4,5,6$ \\
Human & The human users & 3 \\
Power & The power & 2 \\
Sensor & The position sensors & 3 \\
\hline
\end{tabular}

Table 3. Stimuli for the driver side car mirror ECU

\begin{tabular}{|c|c|c|c|c|}
\hline Stimulus & Long Name & Description & Interface & Trace \\
\hline$\overline{\mathrm{MHI}}$ & $\begin{array}{l}\text { Mirror horizontal } \\
\text { inward }\end{array}$ & $\begin{array}{l}\text { Horizontal inward movement of selected } \\
\text { mirror }\end{array}$ & Human & 8 \\
\hline MHO & $\begin{array}{l}\text { Mirror horizontal } \\
\text { outward }\end{array}$ & $\begin{array}{l}\text { Horizontal outward movement of selected } \\
\text { mirror }\end{array}$ & Human & 8 \\
\hline MVD & $\begin{array}{l}\text { Mirror vertical } \\
\text { down }\end{array}$ & $\begin{array}{l}\text { Vertical down movement of selected } \\
\text { mirror }\end{array}$ & Human & 7 \\
\hline MVU & Mirror vertical up & Vertical up movement of selected mirror & Human & 7 \\
\hline PHEI & $\begin{array}{l}\text { Position horizontal } \\
\text { extreme inward }\end{array}$ & $\begin{array}{l}\text { Horizontal position report of driver side } \\
\text { mirror indicating the extreme inward } \\
\text { position is reached }\end{array}$ & Sensor & 8 \\
\hline PHEO & $\begin{array}{l}\text { Position horizontal } \\
\text { extreme outward }\end{array}$ & $\begin{array}{l}\text { Horizontal position report of driver side } \\
\text { mirror indicating the extreme outward } \\
\text { position is reached }\end{array}$ & Sensor & 8 \\
\hline PHNE & $\begin{array}{l}\text { Position horizontal } \\
\text { no extreme }\end{array}$ & $\begin{array}{l}\text { Horizontal position report of driver side } \\
\text { mirror indicating no extreme position is } \\
\text { reached }\end{array}$ & Sensor & 8 \\
\hline PVED & $\begin{array}{l}\text { Position vertical } \\
\text { extreme down }\end{array}$ & $\begin{array}{l}\text { Vertical position report of driver side } \\
\text { mirror indicating the extreme down } \\
\text { position is reached }\end{array}$ & Sensor & 7 \\
\hline PVEU & $\begin{array}{l}\text { Position vertical } \\
\text { extreme up }\end{array}$ & $\begin{array}{l}\text { Vertical position report of driver side } \\
\text { mirror indicating the extreme up posi- } \\
\text { tion is reached }\end{array}$ & Sensor & 7 \\
\hline PVNE & $\begin{array}{l}\text { Position vertical no } \\
\text { extreme }\end{array}$ & $\begin{array}{l}\text { Vertical position report of driver side } \\
\text { mirror indicating no extreme position is } \\
\text { reached }\end{array}$ & Sensor & 7 \\
\hline $\mathrm{SM}$ & Switch mirror & $\begin{array}{l}\text { The switch toggled for selection of driver } \\
\text { side or passenger side mirror }\end{array}$ & Human & 1 \\
\hline START & Start & Car key in start position & Power & 2 \\
\hline
\end{tabular}


Table 4. Responses for the driver side car mirror ECU

\begin{tabular}{|c|c|c|c|c|}
\hline Response & Long Name & Description & Interface & Trace \\
\hline CERR & $\begin{array}{l}\text { CAN mirror movement } \\
\text { failure }\end{array}$ & $\begin{array}{l}\text { Error message generated and put on } \\
\text { CAN bus when mirror is already in } \\
\text { extreme position and cannot make } \\
\text { requested movement. }\end{array}$ & CAN & 9 \\
\hline CMHI & $\begin{array}{l}\text { CAN mirror horizontal } \\
\text { inward }\end{array}$ & $\begin{array}{l}\text { Message is generated and put on CAN } \\
\text { bus for passenger side ECU for hori- } \\
\text { zontal inward movement of passenger } \\
\text { side mirror. }\end{array}$ & CAN & 6,8 \\
\hline $\mathrm{CMHO}$ & $\begin{array}{l}\text { CAN mirror horizontal } \\
\text { outward }\end{array}$ & $\begin{array}{l}\text { Message is generated and put on CAN } \\
\text { bus for passenger side ECU for hori- } \\
\text { zontal outward movement of passenger } \\
\text { side mirror. }\end{array}$ & CAN & 6,8 \\
\hline CMVD & $\begin{array}{l}\text { CAN mirror vertical } \\
\text { down }\end{array}$ & $\begin{array}{l}\text { Message is generated and put on CAN } \\
\text { bus for passenger side ECU for vertical } \\
\text { down movement of passenger side } \\
\text { mirror. }\end{array}$ & CAN & 6,7 \\
\hline CMVU & CAN mirror vertical up & $\begin{array}{l}\text { Message is generated and put on CAN } \\
\text { bus for passenger side ECU for vertical } \\
\text { up movement of passenger side mirror. }\end{array}$ & $\mathrm{CAN}$ & 6,7 \\
\hline $\mathrm{HI}$ & $\begin{array}{l}\text { Horizontal inward move- } \\
\text { ment }\end{array}$ & $\begin{array}{l}\text { Horizontal inward movement of driver } \\
\text { side mirror }\end{array}$ & Actuator & 8 \\
\hline $\mathrm{HO}$ & $\begin{array}{l}\text { Horizontal outward } \\
\text { movement }\end{array}$ & $\begin{array}{l}\text { Horizontal outward movement of } \\
\text { driver side mirror }\end{array}$ & Actuator & 8 \\
\hline VD & Vertical down movement & $\begin{array}{l}\text { Vertical down movement of driver side } \\
\text { mirror }\end{array}$ & Actuator & 7 \\
\hline VU & Vertical up movement & $\begin{array}{l}\text { Vertical up movement of driver side } \\
\text { mirror }\end{array}$ & Actuator & 7 \\
\hline
\end{tabular}

to denote the empty sequence. For our example, the stimuli are alphabetically ordered in Table 3. We concatenate stimuli to string prefixes with periods.

For each sequence one identifies a unique response based on the requirements. For instance, START.PHEI.MHO corresponds to: car key in start position, followed by a horizontal position report indicating that the driver side mirror has reached the extreme inward position, followed by a human command to move the driver side mirror outward. By Requirement 8, the software should produce response HO to make a horizontal outward movement.

When mapping stimulus sequences to responses, there are two special situations. In one case, a sequence generates no externally observable behavior, represented by null (denoted 0 ) in $R$. In the other case, a sequence is physically unrealizable (the sequence cannot happen in practice), hence we introduce another special response illegal (denoted $\omega$ ) into $R$. Therefore, $R$ always contains 0 and $\omega$. A sequence is illegal when it maps to $\omega$; otherwise, it is legal.

For each enumerated sequence $v$, one checks whether $v$ takes the system to a situation encountered with a previous sequence $u$. This is the case if $u$ and $v$, when further extended by any non-empty stimulus sequence $w$, will always generate the same response. For instance, START.MHI and START are two such sequences because we assume (by derived requirement D3 in Table 5. "derived" because there is no justification in the original requirements and hence we document and tag our assumption as a derived requirement) that any mirror adjustment command is ignored before receiving the position report. Two such sequences are Mealy equivalent, as they lead to the same state when the system is 
modeled as a Mealy machine [9]. Note that two Mealy equivalent sequences need not be mapped to the same response but their responses with respect to future extensions always agree. If a sequence is not Mealy equivalent to any previously enumerated sequence, it is unreduced; otherwise, it is reduced to the previously enumerated (Mealy equivalent) sequence that is itself unreduced.

Table 5. Derived requirements for the driver side car mirror ECU

\begin{tabular}{l|l}
\hline Tag & Derived Requirement \\
\hline D1 & It is physically impossible for the ECU to experience an input without power. \\
D2 & $\begin{array}{l}\text { There is no externally observable response across the system boundary when } \\
\text { ignition is turned on. }\end{array}$ \\
D3 & Mirror adjustment commands ignored unless position signal has been received. \\
D4 & No externally observable response when mirror position signal is received. \\
D5 & No externally observable response when the mirror selection switch toggles. \\
D6 & Re-powering on makes previous history irrelevant. \\
D7 & When ignition is turned on, default mirror selection is on the driver side. \\
D8 & When mirror selection switch goes to passenger side, any received or to-be- \\
& received driver side mirror position report will be ignored. Updated position \\
signals are expected once switch goes back to the driver side.
\end{tabular}

One starts with the empty sequence. To get all sequences of length $n+1$ (integer $n \geq 0$ ) one extends all sequences of length $n$ by every stimulus in $S$, and considers the extensions in lexicographic order. This inherently combinatorial process can be controlled by two observations:

- If sequence $u$ is reduced to a prior sequence $v$, there is no need to extend $u$, as the behaviors of the extensions are defined by the same extensions of $v$.

- If sequence $u$ is illegal, there is no need to extend $u$ by any stimulus, as all of the extensions must be illegal (i.e., physically unrealizable).

Therefore, only legal and unreduced (also called extensible) sequences of length $n$ get extended by every stimulus for consideration at length $n+1$. The process continues until all sequences of a certain length are either illegal or reduced to prior sequences. The enumeration becomes complete. This terminating length is discovered in enumeration, and varies from application to application.

Excerpts of an enumeration for the mirror controller is in Table 6] (for the complete enumeration table see [8]). Columns are for sequences, their responses, reductions, and traces to requirements. An unreduced sequence (highlighted) repeats itself in the "Equivalence" column. In constructing the enumeration, we found that supplemental information was needed for enumeration decisions regarding responses and equivalences. We include such information as "derived requirements" in Table 5. They are subject to validation by domain experts. This illustrates one benefit of sequence-based specification, that is, the identification of missing, inconsistent, and incorrect requirements through explicit enumeration.

Application of the method is facilitated with a prototype enumeration tool developed by the Software Quality Research Laboratory (SQRL) at the University 
Table 6. Excerpts of an enumeration without applying string-rewriting

\begin{tabular}{|l|l|l|l|}
\hline Sequence & Response & Equivalence & Trace \\
\hline \hline$\lambda$ & 0 & $\lambda$ & Method \\
\hline MHI & $\omega$ & MHI & D1 \\
\hline \multicolumn{3}{|l|}{$\cdots$} & MHI \\
\hline SM & $\omega$ & START & 2, D2 \\
\hline START & 0 & START & D3 \\
\hline START.MHI & 0 & START & D3 \\
\hline START.MHO & 0 & START & D3 \\
\hline START.MVD & 0 & START & D3 \\
\hline START.MVU & 0 & START.PHEI & D4 \\
\hline START.PHEI & 0 & & \\
\hline \multicolumn{5}{|l|}{$\cdots$} & START.PVNE & D4 \\
\hline START.PVNE & 0 & START.SM & D5 \\
\hline START.SM & 0 & START & 2, D2, D6 \\
\hline START.START & 0 & START.PHEI & 8,9 \\
\hline START.PHEI.MHI & CERR & START & 8 \\
\hline START.PHEI.MHO & HO & START.PHEI & D3 \\
\hline START.PHEI.MVD & 0 & START.PHNE.PVNE & D4 \\
\hline \multicolumn{3}{|l|}{$\cdots$} & ST, D7, D8 \\
\hline START.PHNE.PVNE.PVNE & 0 & START & 2, D2, D6 \\
\hline START.PHNE.PVNE.SM & 0 &
\end{tabular}

of Tennessee [18. To produce a specification in the tool, one only needs to give stimuli and responses short names to facilitate enumeration; no other notation or syntax is required. The tool enforces enumeration rules by the recommended workflow and maintains internal files (XML format) current with every action. The mirror control example was produced using the tool.

\section{Applying String-Rewriting to Sequence Enumeration}

Deciding that two sequences are Mealy equivalent is not always easy. If one could document any observed patterns (for instance, some pairs of inputs commute; some are idempotent), and automatically apply these patterns when they are seen again, it would reduce human effort and also enforce consistent decisions throughout. We find that string-rewriting theory can be applied for this purpose.

\subsection{Assumptions}

By "enumeration" we refer to a, possibly partial, enumeration work product following the process described in Section 2, It corresponds to a table mapping stimulus sequences to responses, equivalent sequences, and traces. Sequences are listed in increasing order of length, and within the same length lexicographically. 
All rows are completely filled in. Table 6 shows excerpts of a complete enumeration table with 217 sequences. If the sequences have rows labeled 0 to 216, any subtable with the rows from 0 to $i, 0 \leq i \leq 216$, is an enumeration.

We assume that we are always working with a minimal enumeration: no equivalence declarations have been missed. This is quite a strong assumption, but a starting point for our theoretical framework. We have also developed a theory that removes this assumption but it is outside of the scope of this paper.

\subsection{Prefix Rewrite Rules}

An enumeration is a possibly partial specification product. The reductions among enumerated sequences automatically define a set of prefix rewrite rules. If sequence $u$ is reduced to prior sequence $v$, then $u$ and $v$ take the system to the same state from the initial state. For any $w \in S^{*}$, the state reached by $u w$ is the same as that reached by $v w$. Hence we rewrite $u w$ to the smaller sequence $v w$ in length-lexicographic order representing the same state. We denote this prefix rewrite rule by $u \models v$, where $\models$ denotes the prefix-rewriting relation.

The first illegal sequence $u$, if there are any, also defines prefix rewrite rules. If we extend $u$ by any stimulus $x \in S, u x$ and $u$ must take the system to the same state. We can rewrite any sequence that contains $u x$ as a prefix to a shorter sequence by replacing $u x$ with $u$. We have one such prefix rewrite rule for each stimulus $x$ in $S$, denoted by $u x \models u$. To summarize, the following prefix rewrite rules are defined for an enumeration with stimulus set $S$ :

- If sequence $u$ reduces to a prior sequence $v$, then $u \models v$.

- If sequence $u$ is the first illegal sequence, then $u x \models u$ for all $x$ in $S$.

As an example, if we enumerate as in Table 6 and stop at Length 2, the following are defined: $x \models$ MHI, where $x$ is any stimulus except MHI; START. $v \models$ START, where $v$ is any stimulus in $\{$ MHI, MHO, MVD, MVU, START $\}$; MHI. $x \models$ MHI, where $x$ is any stimulus in Table 3 .

A reduction system has two parts, a set and a binary relation called the reduction relation on that set [3]. An enumeration with stimulus set $S$ and prefix rewrite rules defined by (1) implies a reduction system: $S^{*}$ is the set, and the reduction relation $\rightarrow$ on $S^{*}$ is defined by $u \rightarrow v$ if and only if there exists $w \in S^{*}$ such that $u=l w, v=r w$ and $l \models r$ is a prefix rewrite rule. If no prefix rewrite rules apply to sequence $u$ then $u$ is said to be a normal form and we showed in [8] (all the conclusions that follow in this paragraph) that otherwise $u$ prefixrewrites to a Mealy equivalent sequence, and that rewriting continues until finally we get to a normal form. Rewriting is guaranteed to terminate, and a unique normal form is obtained for any stimulus sequence. With a completed enumeration, the normal form for every stimulus sequence is the smallest sequence in length-lexicographic order that takes the system to the same state. All sequences that rewrite to the same normal form sequence are Mealy equivalent. 


\section{3 $\quad$ String Rewrite Rules}

A complete enumeration encodes a finite state automaton with Mealy outputs (a Mealy machine). Unreduced sequences represent system states and each row in the enumeration table defines the response and ending state for the transition with a particular stimulus and starting state. For instance, in Table 6 the sequence START.MHI is mapped to 0 and reduced to START. This indicates that from the state represented by unreduced sequence START, the outgoing arc triggered by MHI outputs 0 and loops back into the same state.

The process of deriving an incomplete enumeration could lead to string rewrite rules that reflect general structures of the state machine being identified. These could be exploited later to infer reductions. For example, one might decide that when two position reports in the same axis (horizontal/vertical) are received in a row, the more recent overrides the other. PHEI.PHNE $\vdash$ PHNE is such a rule with $\vdash$ denoting the string rewrite relation. In any sequence one may replace substring PHEI.PHNE (not necessarily as a prefix), with PHNE (hence a string rewrite rule). String rewrite rules can be generalized from already identified sequence reductions. We consider two situations:

- A single reduction suggests a string rewrite rule. Suppose sequence $u$ reduces to the prior sequence $v$. If $u=w_{1} l w_{2}$, and $v=w_{1} r w_{2}$ for some $w_{1}, w_{2} \in S^{*}$, then $l \vdash r$ is a potential string rewrite rule.

- Two reductions suggest a string rewrite rule. Suppose sequences $u$ and $v$ both reduce to prior sequence $w$ ( $w$ is prior to both $u$ and $v$ ) and $u=w_{1} l w_{2}$, $v=w_{1} r w_{2}$ for some $w_{1}, w_{2} \in S^{*}$, then $l \vdash r$ is a potential string rewrite rule.

We require $r$ be length-lexicographically smaller than $l$ for any string rewrite rule $l \vdash r$, so that rewriting is always towards a smaller sequence in lengthlexicographic order for deriving sequence reductions. Also for any $u$ in $S^{*}, u l$ and $u r$ must be Mealy equivalent. To summarize, a string rewrite rule can be defined for an enumeration with stimulus set $S$ as follows:

If the following hold:

- either sequence $u$ reduces to sequence $v$, or sequences $u$ and $v$ reduce to the common prior sequence $w$. In both cases $u=w_{1} l w_{2}$, and $v=w_{1} r w_{2}$ for some $w_{1}, w_{2} \in S^{*}$,

- $r$ is length-lexicographically smaller than $l$,

- $u l$ and $u r$ are Mealy equivalent to each other for all $u$ in $S^{*}$, then $l \vdash r$.

We have seen one rule, PHEI.PHNE $\vdash$ PHNE. Another is PHEI.MHI $\vdash$ PHEI identified when one reduces START.PHEI.MHI to START.PHEI.

An enumeration with string rewrite rules implies a reduction system: $S^{*}$ is the set, and $\rightarrow$ on $S^{*}$ is defined by $u \rightarrow v$ if and only if there exist $w_{1}, w_{2} \in S^{*}$ such that $u=w_{1} l w_{2}, v=w_{1} r w_{2}$, and $l \vdash r$ is a string rewrite rule. We showed in [8] (all the conclusions that follow in this paragraph) that every stimulus sequence to which string rewrite rules apply string-rewrites to a Mealy equivalent sequence. Rewriting continues until a normal form is reached. Rewriting is guaranteed to terminate, but there may be more than one normal form for a sequence. 


\subsection{Combining Prefix String-Rewriting with String-Rewriting}

When enumerating stimulus sequences we may have prefix rewrite rules defined by (1) and string rewrite rules defined by (2). We can combine these rules in a mixed reduction system as follows. Reduction relation $\rightarrow$ on $S^{*}$ is defined by $u \rightarrow v$ if and only if one or both of the two conditions hold: there exists $w$ and prefix rewrite rule $l \models r$ such that $u=l w$ and $v=r w$; or there exists $w_{1}, w_{2} \in S^{*}$ and string rewrite rule $l \vdash r$ such that $u=w_{1} l w_{2}$ and $v=w_{1} r w_{2}$. If (prefix or string) rewrite rules apply to a sequence $u$ then $u$ rewrites to a Mealy equivalent sequence [8]. Rewriting continues until a normal form is derived. As with stringrewriting, rewriting is guaranteed to terminate, and more than one normal form might be obtained. For an incomplete enumeration, the next sequence to be enumerated in length-lexicographic order must have a unique normal form [8].

The reduction system can be used to predict the reduction of the next sequence to be enumerated based on the prefix and string rewrite rules. If the unique normal form is different than the sequence itself, it suggests a sequence reduction. If the sequence itself and the derived unique normal form are identical, the human specifier takes over and considers possible reduction. This suggests that we can develop enumerations and reduction systems concurrently through the process in Table 7, until a complete enumeration is constructed.

Table 7. Major steps of enumeration process with string-rewriting support

1: Let the enumeration with the stimulus set $S$ contain only the empty sequence $\lambda$, mapped to 0 and unreduced. No rules are defined. The reduction system has an empty set as the reduction relation.

2: Repeat the steps below until the enumeration is complete.

3: Derive the (unique) normal form (say $v$ ) of the next sequence (say $u$ ) to be enumerated in length-lexicographic order in the current enumeration, using the current reduction system.

4: The human specifier defines the response (say $r$ ) of $u$.

5: If $v=u$, then the human specifier redefines $v$ such that $u$ is reduced to $v$ by Mealy equivalence and the enumeration rules. If $u$ cannot be reduced to any prior sequence, let $u$ be unreduced.

6: Let the enumeration contain the sequence $u$. $u$ is mapped to $r$ and is either unreduced, or reduced to $v$ by Step 5 .

7: If the response mapping and the equivalence declaration for $u$ define a prefix rewrite rule $l=r$ by (1), then add to the reduction relation $(l y, r y)$ for all $y \in S^{*}$.

8: If the human specifier identifies string rewrite rule $l \vdash r$ by (2) given the equivalence declaration of $u$, then add to the reduction relation $(x l y, x r y)$ for all $x, y \in S^{*}$.

Suppose we apply the process to the mirror controller (for the complete enumeration see [8]). We continue discovering rewrite rules as we enumerate, extending a reduction system while we extend an enumeration. The rules accumulate until they determine another reduction, then string-rewriting is applied automatically. In this example over half of the reductions for the length-four sequences 
(69 of 108) were through automatic string-rewriting. This accounts for almost a third of reductions $(69 / 217)$ needed for a complete enumeration.

\section{Case Studies}

We implemented the theory in our specification tool [18, and used it to construct the car door mirror example. We observe that once the rewrite rules are discovered, they can be checked against the requirements to see if correct decisions have been made, and against each other to see if such decisions have been made consistently. Table 8 shows the rules grouped by rule structures and semantics.

Table 8. Grouping rewrite rules based on structures and semantics

\begin{tabular}{|c|c|}
\hline I & $\begin{array}{l}S_{6}: \text { PHEI.MHI } \vdash \text { PHEI } \\
S_{35}: \text { PVED.MVD } \vdash \text { PVED }\end{array}$ \\
\hline \multirow{11}{*}{ II } & $S_{11}:$ PHEI.PHEO $\vdash$ PHEO \\
\hline & $S_{12}:$ PHEI.PHNE $\vdash$ PHNE \\
\hline & $S_{20}:$ PHEO.PHEO $\vdash$ PHEO \\
\hline & $S_{29}:$ PHNE.PHEO $\vdash$ PHEO \\
\hline & $S_{30}:$ PHNE.PHNE $\vdash$ PHNE \\
\hline & $S_{41}:$ PVED.PVEU $\vdash$ PVEU \\
\hline & $S_{52}:$ PVEU.PVED $\vdash$ PVED \\
\hline & $S_{54}:$ PVEU.PVNE $\vdash$ PVNE \\
\hline & $S_{65}:$ PVNE.PVEU $\vdash$ PVEU \\
\hline & $x . y \vdash y$, where $x, y \in\{$ PHEI, PHEO, PHNE $\}$ \\
\hline & $x . y \vdash y$, where $x, y \in\{\mathrm{PVED}, \mathrm{PVEU}, \mathrm{PVNE}\}$ \\
\hline \multirow{4}{*}{ III } & $S_{13}:$ PHEI.SM $\vdash$ SM \\
\hline & $S_{31}:$ PHNE.SM $\vdash \mathrm{SM}$ \\
\hline & $S_{55}: \mathrm{PVEU} . \mathrm{SM} \vdash \mathrm{SM}$ \\
\hline & $x . \mathrm{SM} \vdash \mathrm{SM}$, where $x \in\{\mathrm{PHEI}, \mathrm{PHEO}, \mathrm{PHNE}, \mathrm{PVED}, \mathrm{PVEU}, \mathrm{PVNE}\}$ \\
\hline \multirow{6}{*}{ IV } & $S_{37}:$ PVED.PHEI $\vdash$ PHEI.PVED $\quad S_{38}:$ PVED.PHEO $\vdash$ PHEO.PVED \\
\hline & $S_{39}:$ PVED.PHNE $\vdash$ PHNE.PVED $S_{49}:$ PVEU.PHEI $\vdash$ PHEI.PVEU \\
\hline & $S_{50}:$ PVEU.PHEO $\vdash$ PHEO.PVEU $S_{51}:$ PVEU.PHNE $\vdash$ PHNE.PVEU \\
\hline & $S_{61}:$ PVNE.PHEI $\vdash$ PHEI.PVNE $\quad S_{62}:$ PVNE.PHEO $\vdash$ PHEO.PVNE \\
\hline & $S_{63}:$ PVNE.PHNE $\vdash$ PHNE.PVNE \\
\hline & $x . y \vdash y . x$, where $x \in\{\mathrm{PVED}, \mathrm{PVEU}, \mathrm{PVNE}\}, y \in\{\mathrm{PHEI}, \mathrm{PHE}$ \\
\hline \multirow{3}{*}{$\mathrm{V}$} & $S_{70}: \mathrm{SM} . \mathrm{MHO} \vdash \mathrm{SM}$ \\
\hline & $S_{71}: \mathrm{SM} . \mathrm{MVD} \vdash \mathrm{SM}$ \\
\hline & SM. $x \vdash$ SM, where $x \in\{$ MHI, MHO, MVD, MVU $\}$ \\
\hline
\end{tabular}

One might further identify patterns. For example, Group II shows a decision regarding receiving consecutive position reports in the same axis (horizontal or vertical). Only the latest report is important (this can be validated by domain experts). Since there are three reports for each axis, the number of such rules is $3 \times 3 \times 2=18$. The fact that Group II contains all the 18 rules demonstrates that this decision has been made consistently throughout the enumeration. 
We can also augment our theory and discover more string rewrite rules by taking into consideration sequences that do not show up in the enumeration table, or sequences with specific patterns. Details are not discussed here due to the page limit (interested readers should see [8]). With these observations almost $90 \%$ of the length-four sequences and half of the total reductions would be derived automatically by string-rewriting. Table 9 shows the statistics.

Table 9. Percentages of automatic sequence reductions by applying rewriting

\begin{tabular}{l|l|l}
\hline & With presented theory & $\begin{array}{l}\text { With presented theory } \\
\text { and observations }\end{array}$ \\
\hline Length-four sequences only & $69 / 108 \approx 63.9 \%$ & $96 / 108 \approx 88.9 \%$ \\
All sequences in the enumeration & $69 / 217 \approx 31.8 \%$ & $103 / 217 \approx 47.5 \%$ \\
\hline
\end{tabular}

Table 10 shows for the car mirror ECU and each enumeration length: the number of sequences extended from the previous length, the number of sequences analyzed, as well as the potential number of sequences to be considered. For analyzed sequences we also record how many reductions are handled by string-rewriting and by humans. This shows the effectiveness of enumeration in controlling the combinatorial growth of the number of sequences to be examined.

Table 10. Sequences analyzed in the car mirror ECU enumeration

\begin{tabular}{r|r|r|r|r|r}
\hline Length & $\begin{array}{r}\text { Sequences } \\
\text { Extended }\end{array}$ & $\begin{array}{r}\text { Sequences } \\
\text { Analyzed }\end{array}$ & $\begin{array}{r}\text { Reductions } \\
\text { by String- } \\
\text { Rewriting }\end{array}$ & $\begin{array}{r}\text { Reductions } \\
\text { by } \\
\text { Humans }\end{array}$ & $\begin{array}{r}\text { Potential } \\
\text { Sequences }\end{array}$ \\
\hline 0 & 0 & 1 & 0 & 1 & 1 \\
1 & 1 & 12 & 0 & 12 & 12 \\
2 & 1 & 12 & 0 & 12 & 144 \\
3 & 7 & 84 & 7 & 77 & 1,728 \\
4 & 9 & 108 & 96 & 12 & 20,736 \\
\hline total & 18 & 217 & 103 & 114 & 22,621 \\
\hline
\end{tabular}

We also applied the enumeration process to three published applications:

- Satellite Operations Software (SOS): software component of a space vehicle that processes commands from the ground control system and supplies communications between an uplink ground site and a downlink ground site [21]

- Mine Pump Controller Software (MPCS): control software of a mine pump that detects the water level, monitors carbon monoxide, methane and airflow levels, and operates the pump with assistance from human operators [1]

- Weigh-In-Motion Data Acquisition Processor (WIMDAP): software for data acquisition used in a weigh-in-motion distributed system that acquires and processes data from load cells, performs real-time monitoring of the analog weight signal, and communicates asynchronously with the host computer [25] 
We summarize the result in Table 11 (for the enumerations see [16, 22, 26]). The benefit of applying string-rewriting depends on the application and the skill of the analyst. For instance, the small number of automatic reduction derivations for the satellite operations software is because the state machine is essentially a chain with little branching. In any case the discovered rewrite rules help articulate unstated patterns or facts that are implicit in the requirements and provide additional criteria for validating specification decisions to requirements.

Table 11. Sequences analyzed in the three published applications

\begin{tabular}{l|r|r|r}
\hline Application & SOS & MPCS & WIMDAP \\
\hline Number of stimuli & 23 & 10 & 14 \\
Terminating enumeration length & 9 & 5 & 4 \\
Sequences extended & 11 & 22 & 13 \\
Sequences analyzed & 254 & 265 & 219 \\
Reductions by string-rewriting & 17 & 47 & 58 \\
Reductions by humans & 237 & 218 & 161 \\
Potential sequences & $1,883,023,236,984$ & 111,111 & 41,371 \\
\hline
\end{tabular}

\section{Related Work}

Sequence-based specification emerged from the functional treatment of software described by Mills [13 15. The development was most directly influenced by the trace assertion method of Parnas [1,17] and the algebraic treatment of regular expressions by Brzozowski [5]. One primary distinction of sequence-based specification is the constructive process used to discover a state machine model.

Rewriting systems were studied for deterministic versions of the trace assertion method [6, 7, 23, 24]. The free choice of canonical traces in [23,24] (as representatives of equivalence classes), and a prefix-closed set of unreduced sequences by construction for any sequence-based specification manifest in the respective applications of rewriting.

In [23, 24] a general trace rewriting relation is used and modified to address possibly non-terminating rewriting sequences. "Smart trace rewriting" was introduced to avoid unfruitful rewriting steps, resulting in a constrained prefix string-rewriting system that guarantees a unique normal form for every string.

Trace rewriting systems [6, 7] algorithmically transform any word of a connected semiautomaton to its canonical form. It is shown that if one imposes prefix-continuity on the set of canonical words, the constructed prefix stringrewriting system is well-behaved. Prefix-continuous sets include prefix-closed sets as a special case. In a sequence-based specification the set of unreduced sequences is prefix-closed by construction.

Our work differs from the previous work in that rewriting techniques are applied to assist in the discovery of a state machine from requirements, and to augment the enumeration process with increased automation. We used unconditional forms of string-rewriting and prefix string-rewriting, and combined them in a mixed reduction system. 


\section{Conclusion}

This paper described a method that used string-rewriting to automate parts of sequence enumeration. Two types of rewrite rules were outlined. Prefix rewrite rules are of the form $l \models r$ for sequences $l$ and $r$. Such a rule says that $l$ and $r$ take the system to Mealy equivalent states when applied in the initial state. Thus, we can rewrite a sequence of the form $u=l w$ to $v=r w$. String rewrite rules generalised this by not requiring the rewriting to occur at the beginning of a sequence. Such a rule $l \vdash r$ allows one to rewrite a sequence of the form $u=w_{1} l w_{2}$ to $v=w_{1} r w_{2}$. Given a set of rewrite rules, it is possible to automatically determine whether a sequence $u$, being considered in sequence enumeration, rewrites to a sequence $v$ previously considered. If this is the case then there is no need to extend $u$ : we know that it reduces to $v$.

The prefix and string rewrite rules initially used will depend on domain knowledge. However, we also discussed heuristics that allow the developer to identify potential additional rules. Automation has the potential to both reduce the cost and time involved in sequence enumeration but also to reduce the scope for human error. In addition to outlining this method, we discussed four case studies. Our results are promising, although the degree to which application of these rewrite rules will expedite the enumeration process will vary with the application. The tool enforces the mathematics but hides the details. The proposed method also provides an opportunity for validation of specification decisions to requirements. This is valuable in offering a new insight or articulating an important fact about the requirements that was unstated.

Current research is focused on other practical matters relevant to the application and development of sequence-based specification. For instance, we expect a thorough treatment of abstractions and abstraction management to produce benefits. As application is usually facilitated by separation of inputs that do not interact (to reduce the size of the input alphabet in an enumeration), composition of sequence-based specifications is also of interest.

\section{References}

1. Bartussek, W., Parnas, D.L.: Using assertions about traces to write abstract specifications for software modules. In: Proceedings of the 2nd Conference of the European Cooperation on Informatics, Venice, Italy, pp. 211-236 (1978)

2. Bauer, T., Beletski, T., Boehr, F., Eschbach, R., Landmann, D., Poore, J.: From requirements to statistical testing of embedded systems. In: Proceedings of the 4th International Workshop on Software Engineering for Automotive Systems, Minneapolis, MN, pp. 3-9 (2007)

3. Book, R.V., Otto, F.: String-Rewriting Systems. Springer, Berlin (1993)

4. Broadfoot, G.H., Broadfoot, P.J.: Academia and industry meet: Some experiences of formal methods in practice. In: Proceedings of the 10th Asia-Pacific Software Engineering Conference, Chiang Mai, Thailand, pp. 49-59 (2003)

5. Brzozowski, J.: Derivatives of regular expressions. Journal of the ACM 11(4), 481-494 (1964) 
6. Brzozowski, J., Jürgensen, H.: Theory of deterministic trace-assertion specifications. Technical report CS-2004-30, University of Waterloo (2004)

7. Brzozowski, J., Jürgensen, H.: Representation of semiautomata by canonical words and equivalences. International Journal of Foundations of Computer Science 16(5), 831-850 (2005)

8. Eschbach, R., Lin, L., Poore, J.H.: Applying string-rewriting to sequence-based specification. Technical report ut-cs-12-692, University of Tennessee, Knoxville (2012), http://web.eecs.utk.edu/ library/TechReports/2012/ut-cs-12-692. pdf

9. Gill, A.: Introduction to the Theory of Finite-State Machines. McGraw-Hill, New York (1962)

10. Hopcroft, P.J., Broadfoot, G.H.: Combining the box structure development method and CSP for software development. Electronic Notes in Theoretical Computer Science 128(6), 127-144 (2005)

11. Joseph, M. (ed.): Real-Time Systems: Specification, Verification and Analysis. Prentice Hall International, London (1996)

12. Lin, L., Prowell, S.J., Poore, J.H.: An axiom system for sequence-based specification. Theoretical Computer Science 411(2), 360-376 (2010)

13. Linger, R.C., Mills, H.D., Witt, B.I.: Structured Programming: Theory and Practice. Addison-Wesley, Boston (1979)

14. Mills, H.D.: Stepwise refinement and verification in box-structured systems. IEEE Computer 21(6), 23-36 (1988)

15. Mills, H.D.: The new math of computer programming. Communications of the ACM 18(1), 43-48 (1975)

16. MPCS: Mine pump controller software enumeration (2012), http://sqrl.eecs.utk.edu/btw/files/MPCS_sr.html

17. Parnas, D.L., Wang, Y.: The trace assertion method of module interface specification. Technical report 89-261, Queens University (1989)

18. Proto_Seq: ESP project (2012), http://sqrl.eecs.utk.edu/esp/index.html

19. Prowell, S.J., Poore, J.H.: Sequence-based software specification of deterministic systems. Software: Practice and Experience 28(3), 329-344 (1998)

20. Prowell, S.J., Poore, J.H.: Foundations of sequence-based software specification. IEEE Transactions on Software Engineering 29(5), 417-429 (2003)

21. Prowell, S.J., Trammell, C.J., Linger, R.C., Poore, J.H.: Cleanroom Software Engineering: Technology and Process. Addison-Wesley, Reading (1999)

22. SOS: Satellite operations software enumeration (2012), http://sqrl.eecs.utk.edu/btw/files/SOS_sr.html

23. Wang, Y., Parnas, D.L.: Simulating the behavior of software modules by trace rewriting. IEEE Transactions on Software Engineering 20(10), 750-759 (1994)

24. Wang, Y., Parnas, D.L.: Simulating the behavior of software modules by trace rewriting. In: Proceedings of the 15th International Conference on Software Engineering, Baltimore, MD, pp. 14-23 (1993)

25. Weigh-In-Motion: Weigh-In-Motion, Cube Management, and Marking User Manual, Oak Ridge National Laboratory, Oak Ridge, TN, Version 0.8.2. (2006)

26. WIMDAP: Weigh-in-motion data acquisition processor enumeration (2012), http://sqrl.eecs.utk.edu/btw/files/WIMDAP_sr.html 